\title{
A NOTE ON THE CONJUGACY OF CARTAN SUBALGEBRAS
}

\author{
DAVID J. WINTER
}

(Received 3 May 1978)

Communicated by D. E. Taylor

\begin{abstract}
The conjugacy of Cartan subalgebras of a Lie algebra $L$ over an algebraically closed field under the connected automorphism group $G$ of $L$ is inherited by those $G$-stable ideals B for which $B / C_{i}$ is restrictable for some hypercenter $C_{i}$ of $B$. Consequently, if $L$ is a restrictable Lie algebra such that $L / C_{i}$ is restrictable for some hypercenter $C_{i}$ of $L$, and if the Lie algebra of Aut $L$ contains ad $L$, then the Cartan subalgebras of $L$ are conjugate under $G$. (The techniques here apply in particular to Lie algebras of characteristic 0 and classical Lie algebras, showing how the conjugacy of Cartan subgroups of algebraic groups leads quickly in these cases to the conjugacy of Cartan subalgebras.)
\end{abstract}

Subject classification (Amer. Math. Soc. (MOS) 1970): 17 B 40, 17 B 45, 20 G 15.

The conjugacy of Cartan subalgebras over algebraically closed fields of characteristic 0 does not carry over to characteristic $p>0$, since there are instances of a Lie algebra having Cartan subalgebras with different dimensions. However, we do have the following theorem for algebraic Lie algebras $\mathbf{G}=$ Lie $G$ over an algebraically closed field $k$ of characteristic $p \geqslant 0$.

TheOREM 1 (Humphreys (1967)). The Cartan subalgebras of the Lie algebra $\mathbf{G}=$ Lie $G$ of a connected algebraic group $G$ are all conjugate under $A d G$.

The purpose of this note is to show that Theorem 1 together with Theorem 2 (below) can be used to establish the conjugacy of Cartan subalgebras of a Lie

This research was supported by the National Science Foundation. 
algebra in a fairly general context which, in particular, covers the cases of Lie algebras of characteristic 0 and classical Lie algebras of characteristic $p>3$.

Throughout the paper, the ground field is an algebraically closed field $k$ of characteristic $p \geqslant 0$.

Definttion 1. A Lie algebra $L$ over $k$ is restrictable if either $p=0$ or $p>0$ and $\left(\operatorname{ad}_{\mathbf{L}} \mathbf{L}\right)^{p \subset} \operatorname{ad}_{\mathbf{L}} \mathbf{L}$. An ideal $\mathbf{B}$ of $\mathbf{L}$ is a restrictable ideal of $\mathbf{L}$ if $\mathbf{B}$ is restrictable as a Lie algebra.

Restrictable Lie algebras are just those Lie algebras which can be given the structure of a restricted Lie algebra (Jacobson (1968)). The condition 'restrictable ideal' used here is much weaker than the condition 'restricted ideal' in restricted Lie algebras.

THEOREM 2 (Winter (1970)). Let $\mathbf{L}$ be a restrictable Lie algebra with restrictable ideal $\mathbf{B}$. Then every Cartan subalgebra of $\mathbf{B}$ is the Fitting null space $\mathbf{B}_{0}(\operatorname{ad}(\mathbf{H} \cap \mathbf{B}))$ in $\mathrm{B}$ of $\mathrm{ad}(\mathrm{H} \cap \mathrm{B})$ for some Cartan subalgebra $\mathrm{H}$ of $\mathrm{L}$.

Defintrion 2. The ith hypercenter $\mathbf{C}_{i}$ of $\mathbf{L}$ is defined recursively by $\mathbf{C}_{0}=\{0\}$ and $\mathbf{C}_{i+1}=\left\{x \in \mathrm{L} \mid[x, \mathbf{L}] \subset \mathbf{C}_{i}\right\}$ for $i=1,2, \ldots$

Proposition 1. Every Cartan subalgebra $\mathbf{H}$ of a Lie algebra $\mathrm{L}$ over $k$ contains all of the hypercenters of $\mathbf{L}$.

Proof. We can assume that the center $C_{1}$ of $\mathbf{L}$ is nonzero (otherwise the hypercenters are all 0 ). Since $H$ contains $C_{1}$ and $H / C_{1}$ is a Cartan subalgebra of $L / C_{1}$ (for example, see Theorem 3 below), $H / C_{1}$ contains the hypercenters of $L / C_{1}$, by induction, so that $\mathbf{H}$ contains the hypercenters of $\mathbf{L}$.

The following theorem, due to Barnes and, later, Block, is proved in Winter (1972), p. 127.

THEOREM 3. Let $\varphi: \mathbf{L}_{1} \rightarrow \mathbf{L}_{2}$ be a surjective homomorphism of Lie algebras over $k$. Then for every Cartan subalgebra $\mathbf{H}$ of $\mathbf{L}_{1}, \varphi(\mathbf{H})$ is a Cartan subalgebra of $\mathbf{L}_{2}$. Moreover, every Cartan subalgebra of $\mathbf{L}_{2}$ is of the form $\varphi(\mathbf{H})$ for some Cartan subalgebra $\mathbf{H}$ of $\mathbf{L}_{\mathbf{1}}$.

THEORem 4. Let $\mathbf{L}$ be a restrictable Lie algebra, $B$ an ideal of $L$ such that $B / C_{i}$ is restrictable for some hypercenter $\mathbf{C}_{i}$ of $\mathbf{B}$. Then if $G$ is a group of automorphisms of $\mathbf{L}$ stabilizing $\mathbf{B}$ and if any two Cartan subalgebras of $\mathbf{L}$ are conjugate under $G$, then any two Cartan subalgebras of $\mathbf{B}$ are conjugate under $G$. 
Proof. Define $\mathbf{L}_{j}, \mathbf{B}_{j}$ recursively by $\mathbf{L}_{0}=\mathbf{L}, \mathbf{B}_{0}=B$ and

$$
\mathbf{L}_{j+1}=\operatorname{ad}_{B_{j}} \mathbf{L}_{j}=\left\{\left.\operatorname{ad} x\right|_{B_{j}} \mid x \in \mathbf{L}_{j}\right\}, \quad \mathbf{B}_{j+1}=\operatorname{ad}_{B_{j}} \mathbf{B}_{j}=\left\{\left.\operatorname{ad} x\right|_{B_{j}} \mid x \in \mathbf{B}_{j}\right\} .
$$

Then $L_{i}$ is restrictable. And, sinee the ideal $B_{i}$ of $L_{i}$ is isomorphic to $B / C_{i}, B_{i}$ is also restrictable. Thus, any Cartan subalgebra of $\mathbf{B}_{i}$ has the form $\mathbf{B}_{i 0}\left(\operatorname{ad}\left(\mathbf{H} \cap \mathbf{B}_{i}\right)\right)$ for some Cartan subalgebra $H$ of $L_{i}$ (Theorem 2). Since any two Cartan subalgebras of $\mathbf{L}_{i}$ are conjugate under the induced action of $G$ on $\mathbf{L}_{i}$ (as one easily sees using Theorem 3), it follows that any two Cartan subalgebras of $\mathbf{B}_{i}$ are conjugate under the induced action of $G$ on $\mathbf{B}_{i}$. But then any two Cartan subalgebras of $\mathbf{B}$ are conjugate under $G$, since the Cartan subalgebras of $\mathbf{B}$ contain $\mathbf{C}_{i}$ (by Proposition 1) and $\mathbf{B}_{i}$ is isomorphic to $\mathbf{B} / \mathbf{C}_{i}$ (see Theorem 3).

COROLlaRY 1. Let $\mathbf{G}=$ Lie $G$ where $G$ is a connected algebraic group. Let $\mathbf{B}$ be an Ad G-stable ideal of $\mathbf{G}$ such that $\mathbf{B} / \mathbf{C}_{i}$ is restrictable for some hypercenter $\mathbf{C}_{i}$ of of $\mathbf{B}$. Then the Cartan subalgebras of $\mathbf{B}$ are conjugate under AdG.

Proor. By Theorem 1, this follows immediately from Theorem 4.

COROLLARY 2. Let $\mathbf{L}$ be a Lie algebra such that ad $\mathbf{L}$ is contained in the Lie algebra of the connected automorphism group $G$ of $\mathbf{L}$. Suppose that $\mathbf{L} / C_{i}$ is restrictable for some hypercenter $\mathbf{C}_{i}$ of $\mathbf{L}$. Then the Cartan subalgebras of $\mathbf{L}$ are conjugate under $G$.

Proof. Apply Corollary 1 to $\mathbf{G}=\mathrm{Lie} G$ and $\operatorname{ad} \mathbf{L}$ to show that the Cartan subalgebras of ad $L$ are conjugate under $\operatorname{Ad} G$, whence the Cartan subalgebras of $\mathbf{L}$ are conjugate under $G$.

The conjugacy of the Cartan subalgebras of a Lie algebra of characteristic 0 follows immediately from Corollary 2 .

To illustrate Theorem 4 and to show that it is more general than Theorem 1, we now give a simple proof of the conjugacy of Cartan subalgebras of classical Lie algebras based on Corollary 1.

THEOREM 5 (Seligman (1957)). Let $\mathbf{L}$ be a classical Lie algebra of characteristic $p>3$. Then the Cartan subalgebras of $\mathbf{L}$ are conjugate under the connected automorphism group of $\mathbf{L}$.

Proof. $L$ has the form $L=[G, G] / \mathbf{C}_{\mathbf{1}}$ where $\mathbf{C}_{\mathbf{1}}$ is the center of $[\mathbf{G}, \mathbf{G}]$ (for example see Humphreys (1967), p. 22). Since $L$ is restrictable by Seligman (1967), p. 48, Corollary 1 applies to $B=[G, G]$. Thus, the Cartan subalgebras of $[G, G]$ are conjugate under $G$, whence the Cartan subalgebras of $L=[G, G] / C_{1}$ are conjugate under the connected automorphism group of $\mathbf{L}$. 


\section{References}

C. Chevalley (1951), Théorie des groupes de Lie, Tome 2 (Hermann, Paris).

J. Humphreys (1967), Algebraic groups and modular Lie algebras, Memoirs of the Amer. Math. Soc. 71, 1-76.

N. Jacobson (1962), Lie algebras (Interscience, New York).

G. Seligman (1957), Some remarks on classical Lie algebras, J. Math. Mech. 6, 549-558.

G. Seligman (1978), Modular Lie algebras (Ergenbnisse 40, Springer-Verlag, New York).

D. J. Winter (1970), 'Cartan subalgebras of a Lie algebra and its ideals', Pacific J. Math. 33 (2), 537-541.

D. J. Winter (1972), Abstract Lie algebras (Mass. Inst. Tech. Press).

Department of Mathematics

University of Michigan

Ann Arbor, Michigan 48104

U.S.A. 